\title{
On the effect of discrete numbers of stars in chemical evolution models
}

\author{
M. Cerviñoo ${ }^{1,2}$ and M. Mollá ${ }^{3}$ \\ ${ }^{1}$ LAEFF (INTA), Apdo. 50727, 28080 Madrid, Spain \\ 2 IAA (CSIC), Camino Bajo de Huétor 24, 18080 Granada, Spain \\ 3 Departamento de Física Teórica, C-XI, Universidad Autónoma de Madrid, 28049 Cantoblanco, Spain
}

Received 25 February 2002 / Accepted 1 August 2002

\begin{abstract}
We examine the impact of discrete numbers of stars in stellar populations on the results of Chemical Evolution Models. We explore the resulting dispersion in the true yields and their possible relation with the dispersion in observational data based on a Simple Closed-Box model.

In this framework we find that the dispersion is larger for the less evolved or low abundance regions. Thus, the age-metallicity relation may be a tracer of the Star Formation History of our Galaxy. This theoretical dispersion is especially high for the relative abundance $\log (\mathrm{N} / \mathrm{O})$ in regions where the total number of stars created is still low. This may explain part of the scatter in the N/O ratio observed in star forming galaxies.

We have also found a first order theoretical estimation for the goodness of a linear fit of the helium abundance vs. $12+\log (\mathrm{O} / \mathrm{H})$ with values of the regression coefficient between 0.9 and 0.7 (independent of sampling effects).

We conclude that it is necessary to include these sampling effects in a more realistic Chemical Evolution Model in order that such a model reproduces, at the same time, the mean value and the dispersion of observed abundances.
\end{abstract}

Key words. galaxies: abundances

\section{Introduction and motivation}

The modelling of any observable related with the integrated contribution of stellar populations needs to assume an Initial Mass Function (IMF) and a Star Formation History (SFH). The representation of such distributions in the form of analytical expresions allows an easy implementation in any evolutionary model. However, such analytical laws are valid only under the assumption of an infinite number of stars, when sampling effects (i.e. the integer nature of the number of stars) are not relevant. Even in the case of the modelling of a system where the real physical properties are known, sampling effects must be present showing a dispersion of the data around the mean value obtained by the model (see, e.g. Cerviño et al. 2000).

Cerviño et al. (2002), based on the work by Buzzoni (1989), have presented a method to evaluate quantitatively the intrinsic dispersion due to sampling effects in spectrophotometric synthesis models. It is important to remark that the dispersion associated to such sampling effects is indeed observed, and used as a distance indicator, in the form of surface brightness fluctuations in elliptical galaxies (see Buzzoni 1993, for a theoretical study of the subject).

In a next step, Cerviño et al. (2001) applied this formalism to some quantities which are time-integrated to be obtained,

Send offprint requests to: M. Cerviño, e-mail: mcs@laeff.esa.es such as the ejected masses of elements by the stars when they evolve. With that work, it was demonstrated that the estimation of these ejection rates may have a large dispersion, mostly in the first $5 \mathrm{Myr}$ of the evolution of a stellar cluster.

On the other hand, the observational data used in the comparison of Galactic Chemical Evolution Models (GCE) have a very large dispersion: in the Milky Way Galaxy (MWG) there exist regions at a same Galactocentric distance with oxygen abundances differing by 0.2 dex, means dispersions as large as $50 \%$. Such dispersion can be due to observational errors, but also to sampling effects. As an example, a stellar cluster that has transformed gas into stars following a Salpeter IMF in the mass range $120-0.08 M_{\odot}$ in a single burst, will produce $6.8 \times 10^{-3} \mathrm{SN} M_{\odot}^{-1}$. In the galactic context, the most massive OB association known (Cygnus OB2, cf. Knödlseder 2000) has transformed into stars $6.5 \times 10^{4} M_{\odot}$ of gas, assuming the presence of $120 \mathrm{O}$ stars in the association (that is, in fact, an upper limit). A region of such characteristics will produce $440 \pm 20 \mathrm{SN}$ in a $63.8 \%(1 \sigma)$ confidence interval due to the limited number of stars in the region. The formal relative dispersion is around 5\%, which will result in a similar relative dispersion in the observed metallicity of that type of regions. So, if the chemical evolution of our Galaxy is driven by such a type of massive stellar clusters, at least, a theoretical relative dispersion around 5\% must be expected in the comparison of GCE models with observed data. But in fact, the dispersion must be 
larger as long as most of the observed OB associations have a lower amount of gas transformed into stars and the enrichment depends on the initial mass of the exploding star.

Taking into account the dispersion found for the stellar yields, we think that the same sampling effect may also produce a dispersion in the interstellar medium abundances. However, the stellar yields do not give directly the abundances in a region. It is necessary to use them as the input in a chemical evolution model. Chemical evolution models are usually computed through numerical methods which solve a system of equations where the IMF such as the SFH are taken into account. However, before computing the dispersion of the abundances predicted in these numerical chemical evolution models, we would like to estimate the importance of these sampling effects with simpler chemical evolution models, and see if the resulting dispersion is at least of a similar order of magnitude to that observed. Therefore, the objective of this paper is to obtain a first order estimation of how relevant these sampling effects can be in simple GCE models in order to consider whether it is necessary to include them in more complicated numerical chemical evolution models.

The structure of the paper is the following: In Sect. 2 we show the application of the formalism presented in Cerviño et al. (2002) to the computation of yields in GCE models. In Sect. 3 we obtain the dispersion on the true yields for several metallicities and turn-off masses. In Sect. 4 we apply the formalism to a GCE closed model and we obtain a first order approximation of the relevance of sampling effects in the results. We show the conclusions and implications of this work in 5.

\section{The computation of stellar ejection rates including sampling effects}

Let us assume a system where, at a given time $t$, there are $n_{\mathrm{d}}\left(m_{i}, t\right)=n_{\mathrm{d}, i}$ stars of a given initial mass $m_{i}$ which have completely evolved and $n_{\mathrm{a}}\left(m_{j}, t\right)=n_{\mathrm{a}, j}$ stars of a given initial mass $m_{j}$ which are still present in the system. The total number of completely evolved stars, $N_{\mathrm{d}}^{\mathrm{Tot}}(t)$, and of stars still present in the cluster, $N_{\mathrm{a}}^{\mathrm{Tot}}(t)$, are:

$N_{\mathrm{d}}^{\mathrm{Tot}}(t)=\sum_{i=1}^{I_{\mathrm{d}}(t)} n_{\mathrm{d}, i}(t) ; \quad N_{\mathrm{a}}^{\mathrm{Tot}}(t)=\sum_{i=1}^{I_{\mathrm{a}}(t)} n_{\mathrm{a}, i}(t)$,

where $I_{\mathrm{d}}(t)$ and $I_{\mathrm{a}}(t)$ are the number of initial masses considered and $n_{\mathrm{d}, i}(t)$ and $n_{\mathrm{a}, i}$ must be integer numbers.

Let us assume that each star of mass $m_{i}$ is able to eject an amount of freshly synthesized element $Z$ given by $m y_{Z}\left(m_{i}\right)=$ $m y_{Z, i}$ and defined as:

$m y_{Z, i}=m(Z)_{\text {ejected }, i}-z\left(m_{i}-m_{\mathrm{rem}, i}\right)$

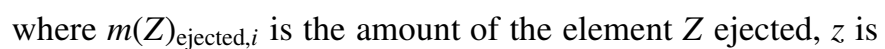
the mass fraction of element $Z$ in the star at the beginning of its evolution and $m_{\mathrm{rem}, i}$ is the mass of the stellar remnant of a star with initial mass $m_{i}$ at the end of its evolution.
The amount of freshly mass of the element $Z$ ejected into the interstellar medium at the time $t, Y_{Z}(t)$ is the sum over all the evolved stars of the individual contribution of each star:

$Y_{Z}(t)=\sum_{i=1}^{I_{\mathrm{d}}(t)} n_{\mathrm{d}, i}(t) m y_{Z, i}$

The corresponding variance of $Y_{Z}(t), \sigma^{2}\left(Y_{Z}(t)\right)$, is the sum of the individual variances. Applying Poisson statistics $\left(\sigma^{2}\left(n_{\mathrm{d}, i}\right)=\right.$ $n_{\mathrm{d}, i}$ ) we obtain:

$\sigma^{2}\left(Y_{Z}\right)=\sum_{i=1}^{I_{\mathrm{d}}} \sigma^{2}\left(n_{\mathrm{d}, i}\right)\left(m y_{Z, i}\right)^{2}=\sum_{i=1}^{I_{\mathrm{d}}} n_{\mathrm{d}, i}\left(m y_{Z, i}\right)^{2}$.

The amount of material locked into stars and stellar remnants is:

$M_{\mathrm{lock}}(t)=\sum_{i=1}^{I_{\mathrm{d}}(t)} n_{\mathrm{d}, i}(t) m_{\mathrm{rem}, i}+\sum_{j=1}^{J_{\mathrm{a}}(t)} n_{\mathrm{a}, j}(t) m_{j}$.

The corresponding variance $\sigma^{2}\left(M_{\text {lock }}(t)\right)$ is:

$\sigma^{2}\left(M_{\mathrm{lock}}(t)\right)=\sum_{i=1}^{I_{\mathrm{d}}(t)} n_{\mathrm{d}, i}(t) m_{\mathrm{rem}, i}^{2}+\sum_{j=1}^{J_{\mathrm{a}}(t)} n_{\mathrm{a}, j}(t) m_{j}^{2}$.

The yield of the element $Z, P_{Z}(t)$, is defined as the fraction of the amount of ejected material over the amount of material locked into stars and stellar remnants:

$P_{Z}(t)=\frac{Y_{Z}(t)}{M_{\text {lock }}(t)}$.

It has an associated variance:

$\sigma_{P_{Z}}^{2}=\frac{\sigma^{2}\left(Y_{Z}\right)}{M_{\text {lock }}^{2}}+\frac{Y_{Z}^{2} \sigma^{2}\left(M_{\text {lock }}\right)}{M_{\text {lock }}^{4}}-2 \frac{Y_{Z}}{M_{\text {lock }}^{3}} \operatorname{cov}\left(M_{\text {lock }}, Y_{Z}\right)$,

where $\operatorname{cov}\left(M_{\text {lock }}, Y_{Z}\right)$ is the covariance:

$\operatorname{cov}\left(M_{\mathrm{lock}}, Y_{Z}\right)=\sum_{i=1}^{I_{\mathrm{d}}} n_{\mathrm{d}, i} m y_{r m Z, i} m_{\mathrm{rem}, i}$

So, at this moment we have the basic ingredients for a GCE model and their corresponding variances. The IMF, $\Phi(m)$ and the SFH, $\Psi(t)$, are implicitly assumed in the computation of the $n_{i}(t)$ values and so, the yield. Since our objective is obtain a first order estimation of the relevance of the discrete number of stars (i.e. sampling effects), we will only examine the case of the true yields.

\section{True yields}

The true yield is defined as the resulting yield of a single stellar population. It is in fact, the yield obtained from a generation of stars that have suffered a single Instantaneous Burst of star formation: $\Psi(t)=M_{\mathrm{s}} \times \delta(t)$ where $M_{\mathrm{s}}$ is the amount of gas that has been transformed into stars in the IMF mass range $m_{\text {up }}$ and $m_{\text {low }}$. In this situation the values of $n_{i}(t)$ can be obtained by a direct integration of the IMF:

$N_{\mathrm{d}}^{\mathrm{Tot}}(t)=M_{\mathrm{S}} \int_{m_{\tau}(t)}^{m_{\mathrm{up}}} \Phi(m) \mathrm{d} m=M_{\mathrm{S}} A \int_{m_{\tau}(t)}^{m_{\mathrm{up}}} m^{\alpha} \mathrm{d} m$ 
where $m_{\mathrm{up}}$ is the upper limit of the IMF, $m_{\tau}(t)$ is the initial mass of the star that ends its evolution at time $t$, that can be approximated by a power law ${ }^{1}: m_{\tau}(t)=B t^{-\gamma}$. The IMF has been normalized to $1 M_{\odot}$ with limits $m_{\text {up }}$ and $m_{\text {low }}$ with a normalization constant $A$. It has been assumed a power-law with slope $\alpha$ over all the mass range. Using this approximation, $N_{\mathrm{d}}^{\text {Tot }}$ has the functional form:

$N_{\mathrm{d}}^{\mathrm{Tot}}(t)=\frac{M_{\mathrm{s}} A}{\alpha+1}\left(m_{\mathrm{up}}^{\alpha+1}-\left(B t^{-\gamma}\right)^{\alpha+1}\right)$.

Let us assume that $m y_{Z, i}$ has a functional form $m y_{Z}(m)=a m+b$. In this case, $Y_{Z}(t)$ and $\sigma^{2}\left(Y_{Z}(t)\right)$ will have the functional form:

$$
\begin{aligned}
Y_{Z}(t)= & M_{\mathrm{s}} \int_{m_{\tau}(t)}^{m_{\mathrm{up}}} \Phi(m)(a m+b) \mathrm{d} m \\
= & \frac{M_{\mathrm{s}} A a}{\alpha+2}\left(m_{\mathrm{up}}^{\alpha+2}-\left(B t^{-\gamma}\right)^{\alpha+2}\right) \\
+ & \frac{M_{\mathrm{s}} A b}{\alpha+1}\left(m_{\mathrm{up}}^{\alpha+1}-\left(B t^{-\gamma}\right)^{\alpha+1}\right) \\
\sigma^{2}\left(Y_{Z}(t)\right)= & M_{\mathrm{s}} \int_{m_{\tau}(t)}^{m_{\mathrm{up}}} \Phi(m)(a m+b)^{2} \mathrm{~d} m \\
= & \frac{M_{\mathrm{s}} A a^{2}}{\alpha+3}\left(m_{\mathrm{up}}^{\alpha+3}-\left(B t^{-\gamma}\right)^{\alpha+3}\right) \\
& +\frac{M_{\mathrm{s}} A 2 a b}{\alpha+2}\left(m_{\mathrm{up}}^{\alpha+2}-\left(B t^{-\gamma}\right)^{\alpha+2}\right) \\
& \times \frac{M_{\mathrm{s}} A b^{2}}{\alpha+1}\left(m_{\mathrm{up}}^{\alpha+1}-\left(B t^{-\gamma}\right)^{\alpha+1}\right) .
\end{aligned}
$$

In the same way, if we assume that $m_{\mathrm{rem}}(m)$ has also a functional form: $m_{\mathrm{rem}}(m)=\mathrm{cm}+d$, we may compute easily $M_{\text {lock }}$ and $\sigma^{2}\left(M_{\text {lock }}\right)$. Both turn out to be functions depending on $m_{\text {up }}$, $m_{\text {low }}$ and $t$.

Let us now show how the relative dispersion in the different quantities scales. At this point it is useful to define it in terms of $\mathcal{N}=N_{\text {eff }}$. This quantity, already used in previous works of this series (see also Buzzoni 1989), is defined, for any quantity $A$ as:

$\frac{\sigma_{A}}{A}=\frac{1}{\sqrt{\mathcal{N}(A)}}$

It means that the larger $\mathcal{N}$, the lower the relative dispersion. In our case:

$$
\begin{aligned}
& \frac{\sigma\left(Y_{Z}\right)}{Y_{Z}} \propto \frac{1}{\sqrt{M_{\mathrm{s}}}} \text { and } \mathcal{N}\left(Y_{Z}\right) \propto M_{\mathrm{s}} \\
& \frac{\sigma\left(M_{\text {lock }}\right)}{M_{\text {lock }}} \propto \frac{1}{\sqrt{M_{\mathrm{s}}}} \text { and } \mathcal{N}\left(M_{\text {lock }}\right) \propto M_{\mathrm{s}}
\end{aligned}
$$

and for the case of the true yield $p_{Z}=Y_{Z} / M_{\text {lock }}$ it can be demonstrated that (see Appendix in Cerviño et al. 2002, for details):

$$
\frac{\sigma\left(p_{Z}\right)}{p_{Z}} \propto \frac{1}{\sqrt{M_{\mathrm{s}}}} \text { and } \mathcal{N}\left(p_{Z}\right) \propto M_{\mathrm{s}} .
$$

\footnotetext{
${ }^{1}$ However, see Cerviño et al. (2001) for remarks in this approximation.
}

Note that $\mathcal{N}$ has the advantage of scaling always with $M_{\mathrm{s}}$ whatever the considered quantities.

With this formalism we have computed the evolution with time of the true yields and the corresponding $\mathcal{N}$ values for hydrogen, helium, carbon, nitrogen and oxygen at five metallicities $(Z=0.020,0.008,0.004$ and 0.0004). We have used the ejected masses presented in Portinari et al. (1998) for massive stars $\left(M>8 M_{\odot}\right)$. These yields include the contribution of stellar winds and those from the type II supernova explosions. For intermediate and low mass stars we have used the yields from Buell (1997), interpolated for the above metallicities, such as those shown in Gavilán et al. (2002). We have considered as solar abundances (in mass) the values $\mathrm{H}=0.735, \mathrm{He}=0.248$, $\mathrm{C}=2.92 \times 10^{-3}, \mathrm{~N}=8.56 \times 10^{-4}$ and $\mathrm{O}=7.95 \times 10^{-3}$ taken from Grevesse \& Sauval (1998). The abundances of carbon, nitrogen and oxygen have been scaled linearly with the metallicity $Z$ for the other metallicities. The helium abundance has been considered as $\mathrm{He}=0.24$ for metallicities different from solar. We had considered a Salpeter IMF slope $(\alpha=-2.35)$ in the mass range 120 to $0.08 M_{\odot}$. Metallicity dependent lifetimes have been taken from Portinari et al. (1998) for all the mass range.

In Fig. 1 we show the evolution of the true yields of $p_{\mathrm{H}}$, $p_{\mathrm{He}}, p_{\mathrm{C}}, p_{\mathrm{O}}$ and $p_{\mathrm{N}}$ and their relative dispersion as a function of time. The relative dispersion has been obtained including the covariance and the dispersion in $M_{\text {lock }}$. However, the values do not differ if it is considered that $M_{\text {lock }}$ has no dispersion at all, except for ages lower than $10 \mathrm{Myr}$. The figure shows that $\mathcal{N}\left(p_{\mathrm{O}}\right)$ reaches a constant value at $\sim 50 \mathrm{Myr}$, when the last supernova explodes $\left(m_{\tau}(t=50 \mathrm{Myr})=8 M_{\odot}\right)$. However, there is an evolution in the $p_{\mathrm{O}}$ value mainly due to the evolution of $M_{\text {lock}}$, that decreases with time, and a small production of oxygen by low mass stars (see below). The values of the true yields $p_{\mathrm{C}}$ and $p_{\mathrm{N}}$ and their associated $\mathcal{N}$ values increase with time. This reflects the contribution of low mass stars to the total yield, which result in an increment on the effective sources that produce these elements and, so, a lower relative dispersion (i.e. larger $\mathcal{N}$ values). The effect is more significant in the case of nitrogen, where the contribution of stars with a lifetime larger than $50 \mathrm{Myr}$ (initial masses lower than $8 M_{\odot}$ ) produces a significant enhancement on the yield. However, the increment is similar for all the considered metallicities, contrary to the expectations for a secondary production of nitrogen. On the other hand, the ejection rates used show an strong dependence of the carbon yield with the initial metallicity, and hence showing a pseudo-secondary behaviour.

We show the evolution of the correlation coefficient of $\rho\left(Y_{\mathrm{H}}, Y_{\mathrm{He}}\right), \rho\left(Y_{\mathrm{H}}, Y_{\mathrm{O}}\right), \rho\left(Y_{\mathrm{He}}, Y_{\mathrm{O}}\right), \rho\left(Y_{\mathrm{O}}, Y_{\mathrm{N}}\right), \rho\left(Y_{\mathrm{O}}, Y_{\mathrm{C}}\right)$, and $\rho\left(Y_{\mathrm{C}}, Y_{\mathrm{N}}\right)$ in Fig. 2. Note that the value of the covariance coefficient is an absolute one, independent of sampling effects. In this case it is a measure of the productions of the elements among different types of stars.

The figure shows that $Y_{\mathrm{H}}$ and $Y_{\mathrm{O}}$, and $Y_{\mathrm{H}}$ and $Y_{\mathrm{He}}$ are almost anti-correlated for all the ages and metallicities. In a first order approximation, it means that the regression coefficient (i.e. the correlation coefficient) of a linear fit of the abundance of helium or oxygen vs. abundance of hydrogen will be close to -1 . In the case of $\rho\left(Y_{\mathrm{He}}, Y_{\mathrm{O}}\right)$ the figure shows that a linear fit of the 

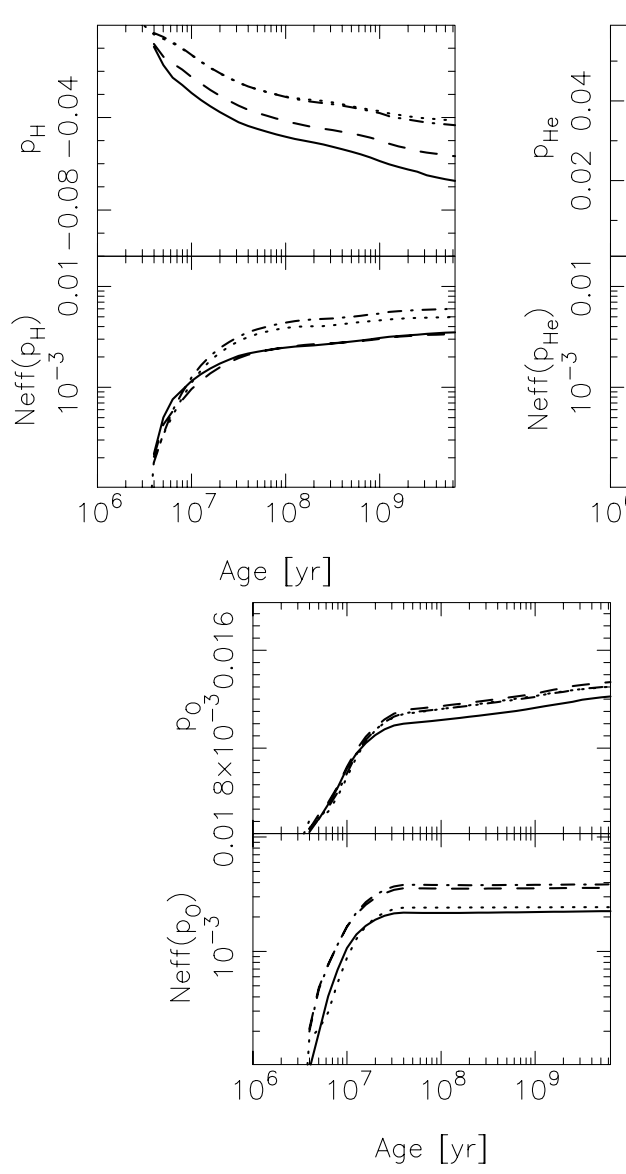
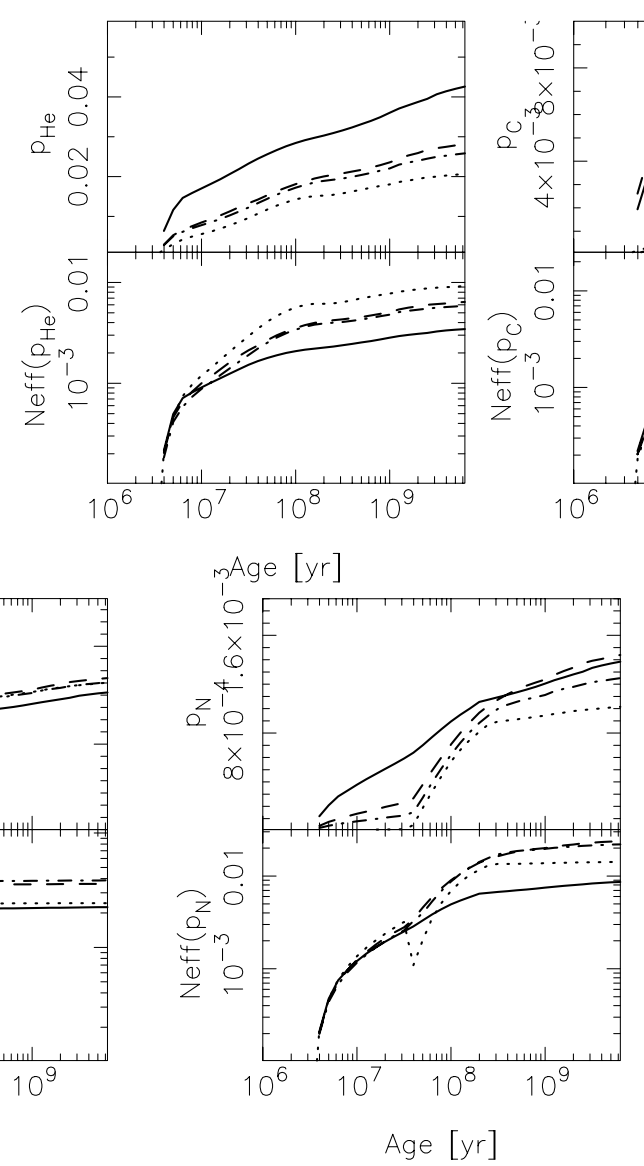

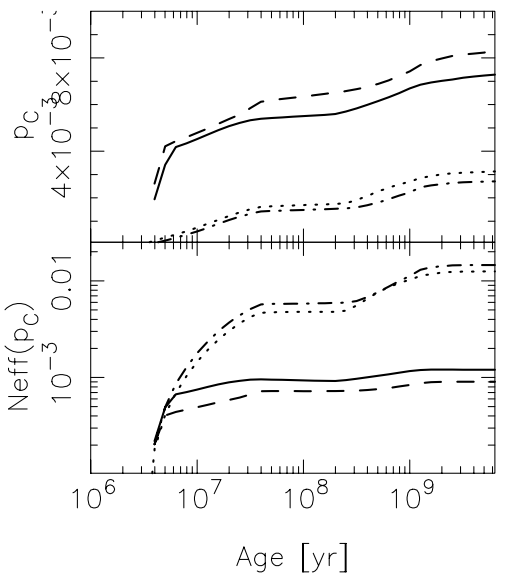

Age $[\mathrm{yr}]$
…. Z $Z=0.0004$

-... Z $Z=0.004$

$-\ldots Z=0.008$

$Z=0.020$

Fig. 1. Evolution of the true yields $p_{\mathrm{H}}, p_{\mathrm{He}}, p_{\mathrm{C}}, p_{\mathrm{O}}$ and $p_{\mathrm{N}}$ and their relative dispersions as a function of time.

helium abundance vs. $12+\log (\mathrm{O} / \mathrm{H})$, as the one used for the determination of the primordial helium abundance, may have a value between 0.9 and 0.7 (i.e. there will be an intrinsic dispersion in such type of representation of the data, as it is indeed observed). However, we want point out that we are working with true yields, which are different from the observed ones.

In the case of $Y_{\mathrm{O}}$ and $Y_{\mathrm{N}}$ the correlation is relatively strong (close to 1) in the first $50 \mathrm{Myr}$, when the production of oxygen and nitrogen is due to a similar population of stars. For ages larger than $50 \mathrm{Myr}$, the correlation coefficient becomes dependent on the metallicity: the lower the metallicity, the closer to zero the correlation coefficient will be. A zero value in the correlation coefficient means that oxygen and nitrogen are produced by different types of stars. It also means, in a first order approximation (but see next section), that the N/O ratio might have a larger dispersion in the low abundance regions, when the contamination of the ISM is due to stars with an initial low metallicity.

The correlation between carbon and oxygen shows a different behavior. The correlation coefficient is also dependent on the initial metallicity, but in this case the lower value is 0.6 , i.e. the production of oxygen and carbon is produced by similar (but not the same) stellar populations. Also, the lower value of the correlation coefficient at solar metallicity implies that, in a first order approximation, the dispersion in the $\mathrm{C} / \mathrm{O}$ ratio will increase with metallicity.
Finally, we had also plotted the correlation between carbon and nitrogen, very similar to the one for oxygen and nitrogen. This is the expected behaviour if carbon and oxygen originate mostly from massive stars. Such a correlation coefficient was also shown in Cerviño et al. (2001) for solar metallicity and ages between 1 and 10 Myr both being quite similar in both cases despite the different treatment used.

Once these true yields, their time evolution when a simple stellar population is created, and their dispersions have been computed, we can use them for calculating the abundances of these elements. As we have already explained, we want to use simple models before treating this subject with numerical models, thus we will use these yields in the most simple GCE model: The Closed-Box Model.

\section{The Closed-Box model}

The original formalism of the Closed-Box model was established by Tinsley (1980). Maeder (1992) revised the formalism and we follow the prescriptions of this author. Let us assume that the total mass in the system, $M$, is constant: $M=s+g=\mathrm{c}$, where $g$ is the amount of mass in the gas and $s$ the amount of mass in stars. In this case

$Z(t)-Z_{0}=p_{Z} \ln \frac{g+s}{g}=p_{Z} \ln \mu^{-1}=-p_{Z}(t) \ln \mu(t)$, 

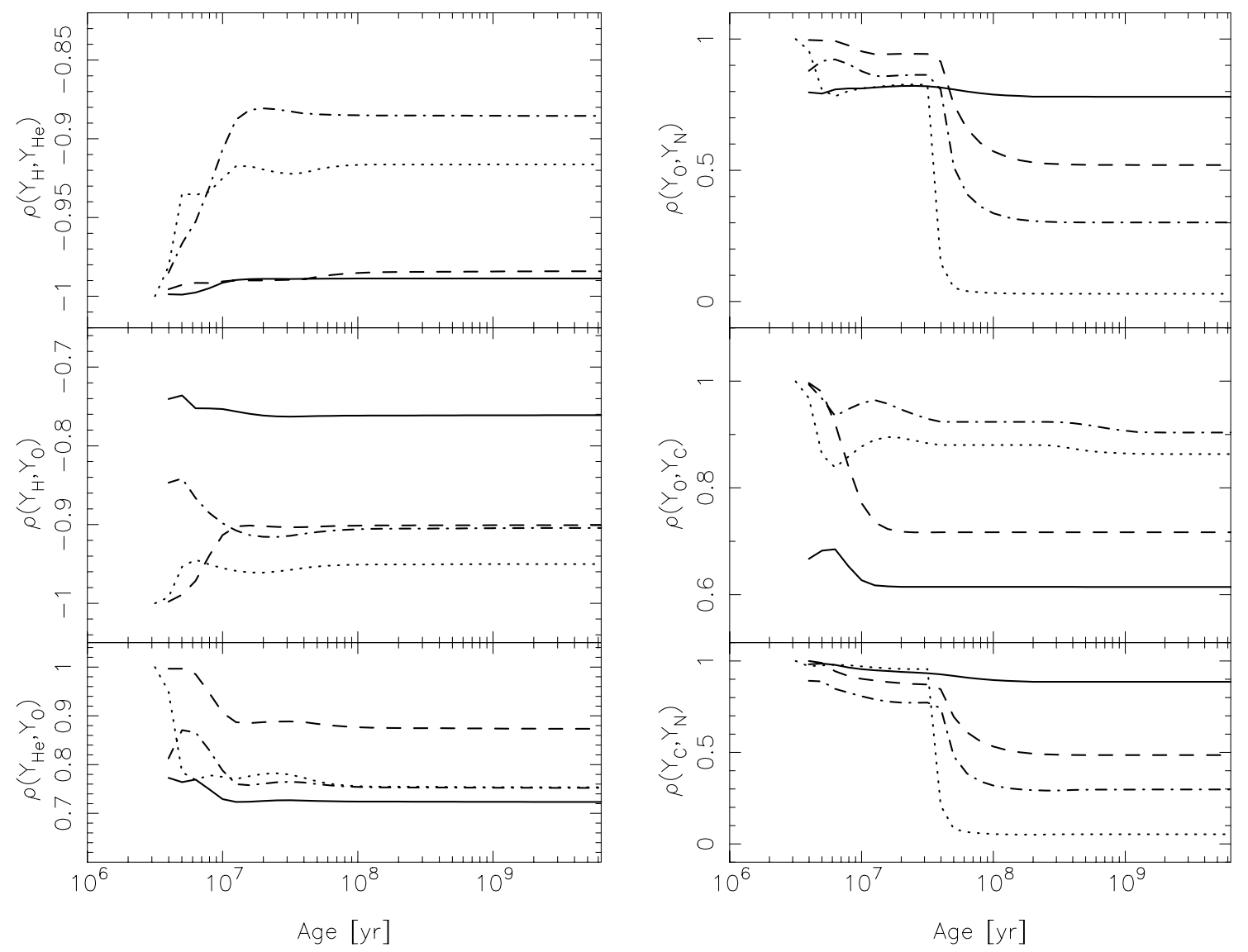

Fig. 2. Evolution of the correlation coefficient $\rho\left(Y_{\mathrm{H}}, Y_{\mathrm{He}}\right), \rho\left(Y_{\mathrm{H}}, Y_{\mathrm{O}}\right), \rho\left(Y_{\mathrm{He}}, Y_{\mathrm{O}}\right), \rho\left(Y_{\mathrm{O}}, Y_{\mathrm{N}}\right), \rho\left(Y_{\mathrm{O}}, Y_{\mathrm{C}}\right)$, and $\rho\left(Y_{\mathrm{C}}, Y_{\mathrm{N}}\right)$ as a function of time. Symbols like in Fig. 1.

where $\mu$ is the gas fraction with an associated variance $\sigma_{\mu}^{2}=$ $\sigma_{\mathrm{s}}^{2} / M^{2}$, and $Z_{0}=Z(t=0)$ is the metallicity at the onset of the burst of star formation.

For simplicity, let us assume that there is only a dispersion due to the discrete number of stars in $Y_{Z}$. In this case, the correlation coefficient $\rho\left(Y_{Z_{1}}, Y_{Z_{2}}\right)$ equals to $\rho\left(p_{Z_{1}}, p_{Z_{2}}\right)$.

The variance in $Z$ is then:

$\sigma_{Z}^{2}=(-\ln \mu)^{2} \sigma^{2}\left(p_{Z}\right)$.

And the relative dispersion of the abundance ratio of two elements $Z_{1}$ respect to $Z_{2}$ is:

$\frac{\sigma_{Z_{1} / Z_{2}}^{2}}{\left(Z_{1} / Z_{2}\right)^{2}}=\ln ^{2} \mu\left[\frac{\sigma_{p_{Z_{1}}}^{2}}{Z_{1}^{2}}+\frac{\sigma_{p_{Z_{2}}}^{2}}{Z_{2}^{2}}-\frac{2 \operatorname{cov}\left(p_{Z_{1}}, p_{Z_{2}}\right)}{Z_{1} Z_{2}}\right]$.

Note that the dispersion scales with $\ln ^{2} \mu$. In a first order approximation it means that the dispersion on ratios of elements will be larger for low metallicity systems.

The Closed-Box Model needs the use of an additional assumption about the true yield. In such model, the time evolution of the true yield is not considered, because, due to the hypotheses involved in its calculation, the true yield corresponds to the total yield obtained for a single stellar generation, that is, the maximum value obtained when all stars die. This is the final value reached at the end of the time evolution in Fig. 1. Thus, the abundance computed with the Closed-Box model is an upper limit of this one which may be actually observed in a star forming region. On other hand the Closed-Box model assumes that all stars are divided in two groups, a first one formed by the low mass stars living forever, and a second one with the more massive which will die immediately after being created. The mass limit between both groups depends on time. Therefore, to use adequately the Closed-Box model, it is necessary define a time scale (or a turn-off mass) $\tau(m)$ where it is assumed that all the stars with lifetimes shorter than $\tau(m)$ (or masses larger than $m$ ) produce all their yield instantaneously. Concerning the total mass transformed into stars, the Closed-Box model is not dependent on the assumed SFH, so, an amount of mass $M_{\mathrm{S}}$ transformed into stars or a constant star formation rate with a value $M_{\mathrm{s}} / \tau(m)$ produce the same result. Finally, it is also needed a value of $\mu$ to describe the evolution of the abundance. In the following we will use a value of $\tau(m)=50 \mathrm{Myr}$ to obtain the dispersion in the age $-\mathrm{O} / \mathrm{H}$ relation and an value of $\tau(m)=1 \mathrm{Gyr}$ to obtain the dispersion on the $\mathrm{N} / \mathrm{O}$ ratio. The metallicity evolution will be performed in terms of $\mu$. Finally, we have assumed the same $Z_{0}$ values than the ones used for the computation of the true yields.

\subsection{Age $-\mathrm{O} / \mathrm{H}$ relation}

Let us now consider the age-metallicity relation. Although this relation is usually given as a function of $[\mathrm{Fe} / \mathrm{H}]$, we will use the abundance $(\mathrm{O} / \mathrm{H})$ as the metallicity indicator. To calculate 

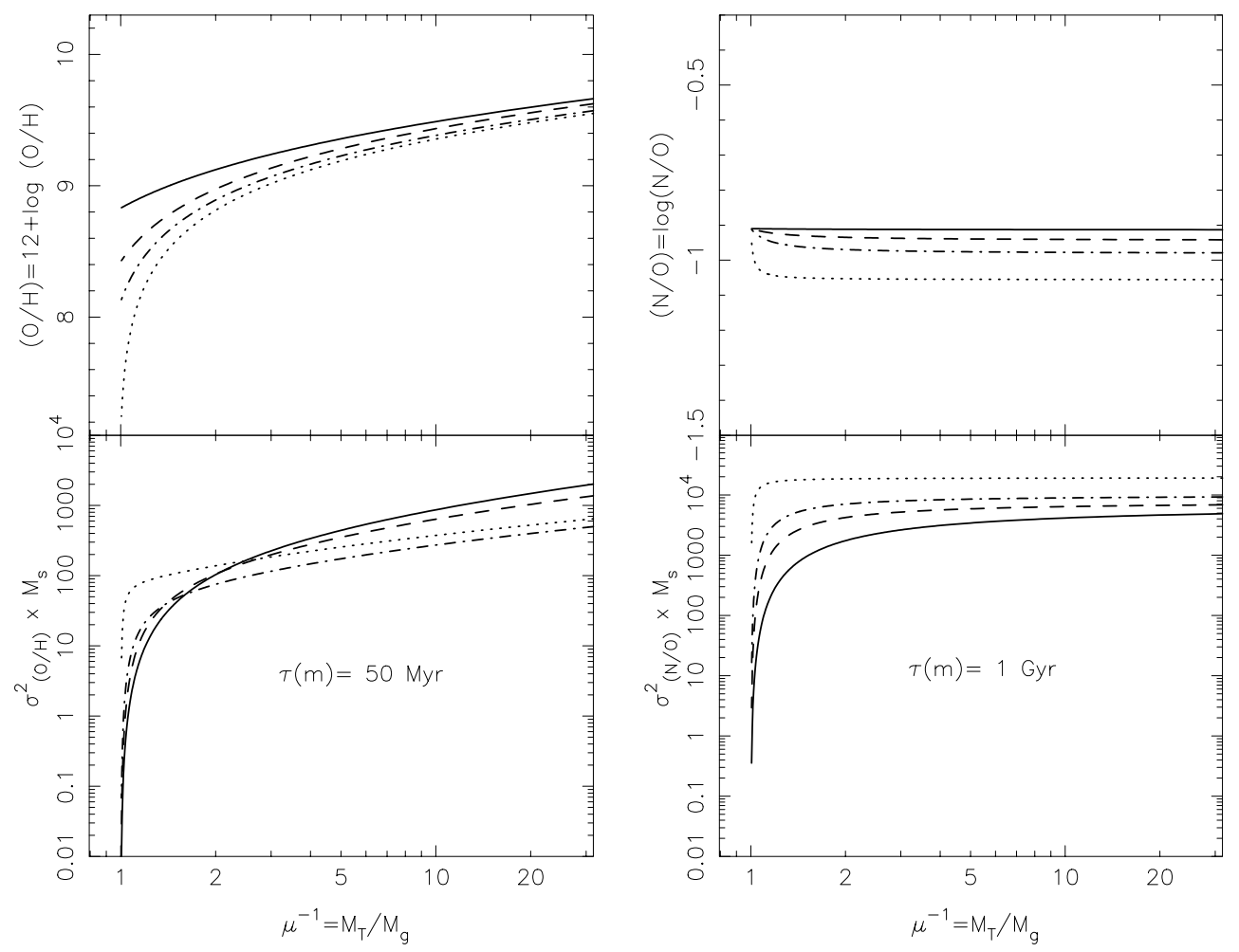

Fig. 3. Evolution of the $12+\log (\mathrm{O} / \mathrm{H})$ and $\log (\mathrm{N} / \mathrm{O})$ ratios as a function of $\mu$, with $\tau(m)=50 \mathrm{Myr}$, for oxygen and $\tau(m)=1 \mathrm{Gyr}$, for nitrogen. Symbols like in Fig. 1.

the iron yield it is necessary to include the ejection from type I SN explosions, which are not considered in this work.

The stellar population has produced most of the oxygen at $50 \mathrm{Myr}$, so we have chosen this value of $\tau(m)$ to obtain the dispersion in the age $-\mathrm{O} / \mathrm{H}$ relation. We have transformed the mass-abundance ratio in the element-abundance ratio by number obtaining this in the used way:

$(\mathrm{O} / \mathrm{H})=12+\log \frac{\mathrm{O}}{\mathrm{H}}=12+\log \frac{Z_{\mathrm{O}}}{16 \times Z_{\mathrm{H}}}$

The corresponding dispersion as a function of $\mu$ is shown in the left panel Fig. 3. The dispersion increases with lower values of $\mu$ as is expected from Eq. (19).

As an example, let us assume the yield at $Z=0.0004$. It reaches a value of $12+\log (\mathrm{O} / \mathrm{H})=8.93$ at $\mu=$ 0.4 . Let us also assume a constant star formation rate of $\Psi(t)=4 M_{\odot} \mathrm{pc}^{-2} \mathrm{Gyr}^{-1}$ for the solar cylinder (cf. Pagel 1977, Table 7.7). In this situation, a dispersion of $\sigma_{(\mathrm{O} / \mathrm{H})}=$ $28.5 \mathrm{pc}^{-1}$ dex is expected, i.e. $\sim 0.3 \mathrm{dex}$ if the star formation takes place in regions of $100 \times 100 \mathrm{pc}^{2}$.

This value is similar to the observational errors. In general, the dispersion will scale with the amount of gas transformed into stars or with the star formation rate (once defined a time scale). So, in this simple framework, the scatter in the agemetallicity relation is related with the Star Formation History itself. Unfortunately, this statement can be only verified with more sophisticated GCE, but that is not the goal of this paper.

It is also interesting to note that the recompilation of correlation coefficients of the fit of $\mathrm{O} / \mathrm{H}$ versus Galactocentric distance for the MWG presented in Henry \& Worthey (1999) has a composite value of -0.63 . In the case of the true yields, the correlation coefficient between $Y_{\mathrm{H}}$ and $Y_{\mathrm{O}}$ varies between $0.75\left(Z=Z_{\odot}\right)$ and $0.95(Z=0.004)$ with a strong dependence on metallicity. In our framework is not possible to compute the correlation coefficient of the relation between $\mathrm{O} / \mathrm{H}$ and the Galactocentric distance, but a more elaborated model must be able to obtain a theoretical value of this correlation coefficient comparable with the observed one.

\subsection{N/O ratio}

For the N/O ratio, we had chosen a value of $\tau(m)=1 \mathrm{Gyr}$ that implies that all the stars with mass larger than $\sim 2 M_{\odot}$ have enriched the ISM with their nitrogen. The N/O ratio has been computed with the relation:

$(\mathrm{N} / \mathrm{O})=\log \frac{\mathrm{N}}{\mathrm{O}}=\log \frac{14 \times Z_{\mathrm{O}}}{16 \times Z_{\mathrm{N}}}$.

The corresponding dispersion as a function of $\mu$ is shown in the right panel of Fig. 3. Again, a direct comparison with observed data can not be performed. First of all, the (N/O) value is fixed by the yields used (and the $\tau(m)$ value). However, it can be shown that a $\sigma_{(\mathrm{N} / \mathrm{O})}$ value larger than 0.5 dex will be a natural effect if the ISM has been contaminated by a burst of star formation with an amount of gas transformed into stars lower than $8 \times 10^{4} M_{\odot}$ or, equivalently, star formation rates lower than $\Psi(t)=80 M_{\odot} \mathrm{Myr}^{-1}$ (using the yield for $Z=$ 0.0004). Again, a more sophisticated model is needed to establish any conclusion. 


\section{Conclusions}

In this work we have shown a theoretical formalism for the evaluation of the dispersion on GCE models. Using a simple Closed-Box model, we have obtained a first order approximation to the relevance of the discreteness of the stellar population and the sampling effects in GCE models. Despite the approximations, the effect of the discreteness of the stellar populations may be relevant for explaining the dispersion in the age-metallicity relation. Such observed dispersion may be an indicator of the SFH of our Galaxy. It may be also relevant in the study of the observed dispersion in the N/O ratio in HII regions and dwarf galaxies.

We have found that the dispersion in the N/O ratio is larger for lower metallicities. This effect is indeed observed in our Galaxy. We have obtained a first order theoretical estimation for the goodness of a linear fit of the helium abundance vs. $12+$ $\log (\mathrm{O} / \mathrm{H})$ with values of the regression coefficient between 0.9 and 0.7 .

Finally, we want to note that in the case of GCE models attempting to trace the metallicity distribution of our Galaxy, the dispersion will not scale with the total mass of the Galaxy. Instead, it must be computed with the masses of the subsystems that are sampled: the dispersion of abundances for individual HII regions from the global metallicity gradient observed in our Galaxy (Henry \& Worthey 1999) could be understood in this framework since these regions are less massive systems.

However, no conclusion can be obtained unless this effect is included in a more sophisticated GCE. It is therefore necessary to make a more exhaustive study on this subject. It will be performed in forthcoming papers.

Acknowledgements. We want to acknowledge Prof. Chiosi and V. Luridiana for their useful suggestions which motivated us to write this paper. We also want to acknowledge V. Luridiana, J. M. Vílchez and L. Carigi for useful discussions.

\section{References}

Buell, J. F. 1997, Ph.D. Thesis, Univ. of Oklahoma, U.S.A Buzzoni, A. 1989, ApJS, 71, 871

Buzzoni, A. 1993, A\&A, 275, 433

Cerviño, M., Luridiana, V., \& Castander, J. F. 2000, A\&A, 360, L5

Cerviño, M., Valls-Gabaud, D., Luridiana, V., \& Mas-Hesse, J. M. 2002, A\&A, 381, 51

Cerviño, M., Gómez-Flechoso, M. A, Castander, F. J., et al. 2001, A\&A, 376, 422

Gavilán, M., Mollá, M., \& Buell, J. 2002, in preparation

Grevesse, N., \& Sauval, A. J. 1998, Space Sci. Rev., 85, 161

Henry, R. B. C., \& Worthey, G. 1999, PASP, 111, 919

Knödlseder, J. 2000, A\&A, 360, 539

Maeder, A. 1992, A\&A, 264, 105

Pagel, B. E. 1997, Nucleosynthesis and Chemical Evolution of Galaxies (Cambridge University Press)

Portinari, L., Chiosi, C., \& Bressan, A. 1998, A\&A, 334, 505

Tinsley, B. M. 1980, Fund. Cosmic. Phys., 5, 287 